\title{
MÉXICO: EL REGRESO DEL PRI A LA PRESIDENCIA
}

\author{
Mexico: The Return of the PRI to the Presidency
}

\author{
JUAN C. OLMEDA \\ Northwestern University
}

\section{MARÍA ALEJANDRA ARMESTO}

Universidad Autónoma Metropolitana

\begin{abstract}
RESUMEN
Presentamos los principales eventos políticos de México durante 2012, año que tuvo como tema central la elección que trajo de regreso al Partido Revolucionario Institucional a la presidencia. Un fenómeno que dejó la campaña presidencial fue la emergencia del movimiento \#YoSoy132, conformado principalmente por jóvenes y universitarios descontentos con la posible vuelta del PRI. La economía continuó su crecimiento lento pero estable, al tiempo que diferentes reformas en materia laboral, educativa y política fueron aprobadas en el Congreso, así como una ley de víctimas. Asimismo, reseñamos la evolución de la violencia -homicidios y desapariciones-así como cambios en el tratamiento de violaciones a los derechos humanos.
\end{abstract}

Palabras clave: México, Elecciones, Peña Nieto, \#YoSoy132, violencia.

\begin{abstract}
We review the main political events in Mexico during 2012. During this year, the election that brought the Partido Revolucionario Institucional back to Mexico's presidency appeared as a central episode. One of the legacies of the presidential campaign was the emergence of the social movement \#YoSoy132 made up primarily of youngsters and university students. During 2012, the Mexican economy continued to grow, slowly but at a steady pace, while different reforms were approved in Congress. We review the labor, educational, and political reforms. Finally, we describe the evolution of violence-homicides and disappearances-and changes in the prosecution of human rights violations.
\end{abstract}

Key words: Mexico, elections, Peña Nieto, \#YoSoy132, violence. 


\section{INTRODUCCIÓN}

Durante 2012, la vida en México giró principalmente en torno a las elecciones del 1 de julio. No solo se eligió a un nuevo presidente, también gobernadores y autoridades locales en varias entidades federativas, y se renovaron por completo las dos cámaras del Congreso. La victoria de Enrique Peña Nieto, candidato por el Partido Revolucionario Institucional (PRI), fue sin duda la noticia central, y supuso el regreso del PRI a la presidencia luego de doce años. El proceso electoral dejó además el surgimiento del movimiento \#YoSoy132, integrado principalmente por jóvenes y universitarios y que adquirió una visibilidad sustantiva durante los meses previos y posteriores a la elección.

Si la primera parte del año estuvo definida por el apronte de las candidaturas y el desarrollo de la campaña electoral, durante la segunda la atención estuvo centrada en la definición del gabinete entrante y en las líneas de acción del nuevo gobierno.

El 2012 estuvo además marcado por la continuidad de la violencia en el marco de la "guerra" contra el narcotráfico que constituyó uno de los pilares de la administración de Calderón. Aun cuando el tema no resultó central durante la campaña electoral, en mayor o menor medida todos los candidatos plantearon la necesidad de redefinir la estrategia en el sexenio que se inicia, y el nuevo presidente ya ha dado pasos en este sentido.

Asimismo, la economía mexicana continuó su crecimiento lento pero estable, a pesar de cierta desaceleración en la economía de Estados Unidos y los efectos en el mercado global de la crisis europea. Dicha evolución favorable estuvo principalmente motivada por el mayor dinamismo del consumo interno, el aumento de las exportaciones no petroleras y el énfasis gubernamental en la estabilidad macroeconómica.

En las secciones que siguen se abordan más en detalle los temas anteriormente mencionados y hacia el final se plantean ciertos escenarios pensando en el futuro próximo.

\section{COYUNTURA POLÍTICA}

\section{Elección presidencial}

La agenda política de México durante 2012 estuvo dominada por la elección presidencial que determinó el regreso del PRI a la presidencia después de doce años de gobierno del PAN. Los candidatos presidenciales fueron cuatro: Enrique Peña Nieto, postulado por una coalición que reunió al PRI y al Partido Verde Ecologista de México (PVEM); Andrés Manuel López Obrador, candidato de una coalición de centro-izquierda formada por el Partido de la Revolución Democrática (PRD), el Partido del Trabajo (PT) y Movimiento Ciudadano (MC); Josefina Vázquez Mota, del centro-derechista PAN; y Gabriel Quadri, postulado por el Partido Nueva Alianza (PANAL), brazo electoral del Sindicato Nacional de Trabajadores de la Educación (SNTE) que encabezaba Elba Esther Gordillo.

Desde el inicio de la campaña en marzo Peña Nieto lideró las preferencias con una intención de voto de entre 39 y 54 por ciento y durante semanas se lo vislumbró como seguro ganador. Su candidatura se apoyó en su imagen personal (construida desde 
sus años como gobernador del estado de México -2005 a 2011- con el apoyo de una poderosa maquinaria mediática) y en las estructuras territoriales y clientelares del PRI. Para ciertos sectores de la sociedad, sin embargo, Peña aparecía asociado al pasado -y presente- autoritario y clientelar del PRI y a la idea de que era un candidato construido desde el marketing, pero en el fondo superficial y vacío. Sus propuestas se centraron en la necesidad de dinamizar la economía a partir de reformas estructurales, y en un cambio en la estrategia de seguridad centrada en la reducción de la violencia.

Si bien Josefina Vásquez Mota inició la campaña en un segundo lugar, contando con la preferencia de entre 20 y 30 por ciento de los votantes, luego del primer debate a mediados de mayo fue desplazada al tercer puesto. Vásquez Mota ganó la candidatura del partido gobernante en una dura contienda interna, y por primera vez en la historia de México uno de los tres principales partidos llevó a una mujer como candidata a la presidencia. Tenía como fortaleza haberse desempeñado como Secretaria de Desarrollo Social durante la presidencia de Fox y de Educación Pública durante la de Calderón, antes de convertirse en líder de la fracción parlamentaria del PAN en la Cámara de Diputados en 2009. Su campaña sin embargo se vio debilitada por una serie de errores en la organización de sus primeros actos y sobre todo por la falta de apoyo de líderes importantes del partido. Su propuesta osciló entre la defensa de la continuidad y la necesidad de diferenciarse de Calderón, destacando temas como la reforma laboral en materia económica y la política de mano dura en lo relativo a seguridad pública y combate al crimen organizado.

López Obrador comenzó la campaña en un lejano tercer puesto, pero a mediados de mayo se consolidó en el segundo lugar alcanzando alrededor del 34 por ciento de la intención de voto. Al acercarse la elección se situaba entre siete y dieciocho puntos porcentuales por debajo de Peña Nieto (Fuentes Berain, 2012). Esta fue su segunda vez como candidato presidencial, luego de perder por menos de un punto frente a Calderón en 2006 y encabezar un largo conflicto post-electoral al denunciar fraude (lo cual lo enfrentó con ciertos sectores de la sociedad mexicana). Sus principales propuestas giraron en torno a la necesidad de superar el modelo económico de las últimas décadas, dando un rol más activo al Estado en la promoción del empleo, el crecimiento y la explotación de los recursos naturales (por ejemplo, expresó su firme oposición a la apertura de PEMEX a la inversión privada). Otra propuesta central fue la revisión de la estrategia punitiva de combate al narcotráfico de Calderón, apuntando al combate a la pobreza y la desigualdad y retirando a las fuerzas armadas de las funciones de seguridad pública.

Gabriel Quadri ocupó durante toda la campaña el cuarto lugar, aun cuando registró un crecimiento en las preferencias luego del primer debate. Quadri intentó presentarse como candidato "independiente" y ciudadano, aunque su mayor debilidad fue ser postulado por un partido asociado a la desprestigiada líder del sindicato de maestros. En verdad, el objetivo de esta candidatura fue obtener al menos el dos por ciento de los votos, mínimo requerido para que el partido mantuviese el registro, y posicionar a esta fuerza como el fiel de la balanza en las negociaciones legislativas.

En una primera fase de la campaña, la ventaja de Peña Nieto lo hizo aparecer como casi seguro ganador. La lenta caída de Vázquez Mota comenzó a dejar en claro que 
la contienda sería entre el priista y López Obrador, quien intentó acortar distancias adoptando un discurso menos confrontativo y radical que en el pasado, resaltando el legado autoritario del PRI y proyectando a Peña Nieto como una mera construcción mediática. Aunque no hubo un tema central durante la campaña, fue llamativo que la cuestión de la violencia no se convirtiera en uno de los ejes del debate. Finalmente, y como se verá más adelante, el evento que sacudió la contienda y puso en duda el triunfo del PRI fue la emergencia del movimiento de jóvenes \#YoSoy132. ${ }^{1}$

Las elecciones discurrieron con normalidad y, a pesar del temor por los altos índices de violencia en el país, no hubo incidentes relevantes. La participación alcanzó un 63 por ciento del padrón electoral y Peña Nieto resultó el candidato más votado con algo más del 38 por ciento de los votos, siete puntos porcentuales por encima de López Obrador. ${ }^{2}$ Este último, sin embargo, no reconoció su derrota y denunció inequidades -compra de votos, gasto excesivo, trato preferencial a favor de Peña Nieto por parte de los medios-, aunque desistió de promover un conflicto post-electoral de la magnitud del de 2006 (Flores-Macías, 2013). Nueva Alianza alcanzó su objetivo de preservar su registro y el PAN fue el gran derrotado logrando apenas un veinticinco por ciento de los votos para la presidencia (Ver Tabla 1).

Tabla 1: Resultados Elección de Presidente 2012

\begin{tabular}{llrc}
\hline \multicolumn{1}{c}{ Partido o Coalición } & \multicolumn{1}{c}{ Candidato } & \multicolumn{1}{c}{ Votos } & Porcentaje \\
\hline PRI-PVEM & Enrique Peña Nieto & 19.226 .784 & 38,21 \\
PRD PT CONV & Andrés Manuel López Obrador & 15.896 .999 & 31,59 \\
PAN & Josefina Vásquez Mota & 12.786 .647 & 25,41 \\
Nueva Alianza & Gabriel Quadri & 1.150 .662 & 2,29 \\
No registrados & & 20.907 & 0,04 \\
Voto Nulo & & 1.241 .154 & 2,47 \\
Total & & 50.323 .153 & 100 \\
\hline
\end{tabular}

Fuente: Instituto Federal Electoral, http://www.ife.org.mx/portal/site/ifev2/Estadisticas_y_ Resultados_Electorales/

La victoria del PRI puede explicarse a partir de tres factores. Primero, la sostenida pérdida de popularidad del presidente Calderón a lo largo de su presidencia, que decididamente afectó las chances de su partido y candidata. En junio de 2012, un mes antes de la elección, Calderón alcanzó el nivel más bajo de todo su sexenio, con sólo $48 \%$ de aprobación (16 puntos porcentuales menos que el pico de popularidad de mayo de 2010). ${ }^{3}$ Para esas fechas, un $44 \%$ de los encuestados se mostraba en desacuerdo con

La Jornada, “Con su actuación, \#YoSoy132 sacó al proceso electoral de una inercia de desinterés: UIA" 4 de septiembre de 2012. http:/ / www.jornada.unam.mx/2012/09/04/politica/008n1pol.

En México, la elección presidencial es por mayoría simple (sin segunda vuelta).

El Universal, “Se desploma 10 puntos la aprobación de Calderón” 5 de junio de 2012. http: / / www.eluniversal. com.mx/notas/851477.html 
la estrategia de lucha contra el narcotráfico y un 56\% desaprobaba la política contra la pobreza. ${ }^{4}$ En segundo lugar, debido a la capacidad de Peña Nieto para evitar divisiones en su partido -en contraste con lo acontecido en 2006- y lograr el apoyo de todas las facciones y de los gobernadores priistas a su candidatura. En tercer lugar, a raíz de la aún existente animadversión de diferentes sectores de la sociedad a la figura de López Obrador, a pesar de los esfuerzos del candidato por moderar sus posturas, lo cual le impidió capitalizar totalmente a su favor el descontento con las administraciones panistas.

Un análisis detallado de los resultados indica que Peña Nieto fue favorecido por los votantes a lo largo y a lo ancho del país, mientras el apoyo a López Obrador y Vásquez Mota estuvo concentrado en los bastiones electorales de sus partidos (Magaloni et al., 2012). Paradójicamente, los votantes más pobres parecen haber hecho una evaluación positiva de las políticas de alivio a la pobreza iniciadas o expandidas durante las dos presidencias panistas -el programa de transferencias condicionadas Oportunidades y el Seguro Popular- y favorecieron con su voto a Vázquez Mota. Sin embargo, el PRI continuó siendo el partido más fuerte entre la población rural y los indígenas (Magaloni et al., 2012).

Por primera vez, las elecciones presidenciales de 2012 estuvieron reguladas por las reformas electorales adoptadas en 2007 y 2008, que en parte respondieron a los alegatos de fraude en 2006 y apuntaron a garantizar equidad y transparencia, y a restablecer la confianza en la autoridad electoral y las elecciones (Casar, 2009). Se estableció que el tiempo de campaña fuera reducido de seis a tres meses, un techo de diez por ciento para el financiamiento privado y una reducción del 48 por ciento al financiamiento público. Además se prohibió la venta de espacios televisivos para propaganda electoral, siendo los tiempos asignados de manera equitativa a los partidos por el Instituto Federal Electoral (IFE) (Langston, 2009). A pesar de los cambios, el proceso de 2012 y sus resultados fueron nuevamente cuestionados, multiplicándose las denuncias acerca de gastos por encima de los límites establecidos por la ley y en relación con la compra y coacción del voto. ${ }^{5}$ En contra de lo pretendido, la confianza de la población en el IFE no solo no mejoró sino que incluso empeoró, pasando de una calificación de 6,9 en 2006 a una de 6,7 en 2012 (Consulta Mitofsky, 2006 y 2012).

\section{Elecciones legislativas}

El 1 de julio los mexicanos también eligieron 500 diputados federales por un período de tres años -300 por el principio de mayoría simple y 200 por el de representación proporcional-y 128 senadores por un período de seis años (tres senadores por cada una de las 32 unidades subnacionales -dos por la mayoría y uno por la primera minoría- y 32 senadores más elegidos por representación proporcional).

4 Excélsior, “La veda golpea la imagen de Calderón” 16 de julio de 2012. http://www.excelsior.com.mx/2012/07/16/ nacional/847915

5 Washington Post, “Mexico's presidential election tainted by claims of vote buying”, 4 de julio de 2012. http:/ / articles.washingtonpost.com/2012-07-04/world/35486148_1_pe-a-nieto-prepaid-gift-cards-election-officials. 
En estas elecciones legislativas el PRI obtuvo la mayor cantidad de votos, aun cuando esto (como se verá más adelante) no sirvió al presidente electo para asegurar una mayoría legislativa propia. Otro hecho significativo fue la caída del PAN, en consonancia con el tercer lugar obtenido en las presidenciales (ver Tabla 2).

Tabla 2: Resultados de las elecciones legislativas federales por partido y/o alianza en 2012

\begin{tabular}{|c|c|c|c|c|c|c|c|c|}
\hline & \multicolumn{4}{|c|}{ Diputados federales } & \multicolumn{4}{|c|}{ Senadores } \\
\hline & \multicolumn{2}{|c|}{ Mayoría relativa } & \multicolumn{2}{|c|}{$\begin{array}{l}\text { Representación } \\
\text { proporcional }\end{array}$} & \multicolumn{2}{|c|}{ Mayoría relativa } & \multicolumn{2}{|c|}{$\begin{array}{c}\text { Representación } \\
\text { proporcional }\end{array}$} \\
\hline & Votos & $\%$ & Votos & $\%$ & Votos & $\%$ & Votos & $\%$ \\
\hline PAN & 12.895 .902 & 25,89 & 12.971 .363 & 25,9 & 13.126 .478 & 26,28 & 13.245 .088 & 26,33 \\
\hline PRI & 15.905 .440 & 31,93 & 15.972 .548 & 31,89 & 15.606 .639 & 31,25 & 15.679 .729 & 31,17 \\
\hline PRD & 9.144 .279 & 18,36 & 9.203 .767 & 18,38 & 9.268 .519 & 18,56 & 9.353 .879 & 18,59 \\
\hline Verde & 3.048 .178 & 6,12 & 3.057.511 & 6,1 & 2.870 .802 & 5,75 & 2.881 .026 & 5,73 \\
\hline PT & 2.288 .894 & 4,59 & 2.296 .461 & 4,59 & 2.326 .920 & 4,66 & 2.339 .923 & 4,65 \\
\hline Convergencia & 1.993 .529 & 4 & 2.001 .951 & 4 & 2.013.706 & 4,03 & 2.025 .045 & 4,03 \\
\hline Nueva Alianza & 2.033 .159 & 4,08 & 2.043.281 & 4,08 & 1.846 .178 & 3,7 & 1.855 .403 & 3,69 \\
\hline $\begin{array}{l}\text { Candidatos } \\
\text { no registrados }\end{array}$ & 35.277 & 0,07 & 35.713 & 0,07 & 32.112 & 0,06 & 32.567 & 0,06 \\
\hline Votos nulos & 2.472 .614 & 4,96 & 2.501 .607 & 4,99 & 2.855 .520 & 5,72 & 2.897 .668 & 5,76 \\
\hline Votación total & 49.817 .272 & 100 & 50.084 .202 & 100 & 49.946 .874 & 100 & 50.310 .328 & 100 \\
\hline
\end{tabular}

Fuente: Instituto Federal Electoral, http://www.ife.org.mx/portal/site/ifev2/

Estadisticas_y_Resultados_Electorales/

Un hecho importante fue que los partidos se vieron obligados a cumplir con la cuota de cuarenta por ciento de candidatas mujeres en sus listas y los resultados de las elecciones dieron lugar a la legislatura con el mayor número de mujeres en la historia: casi treinta y siete por ciento en la Cámara de Diputados y el treinta y tres por ciento en el Senado. ${ }^{6} \mathrm{Si}$ bien las cuotas de género fueron introducidas en México en 1993, los partidos encontraban vías para sortearlas debido a los débiles mecanismos para garantizar su cumplimiento. Las cuotas se cubrían incluyendo mujeres en las candidaturas suplentes o en las posiciones más rezagadas en las listas de representación proporcional; exceptuando del requisito de las cuotas las candidaturas definidas por elecciones internas; o bien, como en 2009, completando la lista con candidatas que pedían licencia luego de asumir dejando en su lugar a suplentes hombres.

De cara a 2012, un grupo de mujeres militantes de los partidos Movimiento Ciudadano, Nueva Alianza y el PRI presentó un juicio ciudadano, y el Tribunal Electoral del Poder

6 En la legislatura anterior las mujeres representaban el 31 por ciento en la Cámara de Diputados y sólo el 14 por ciento en el Senado. 
Judicial de la Federación emitió a fines de 2011 una resolución ordenando a los partidos respetar la cuota de género en sus candidaturas legislativas. Al momento de registrar las listas de candidatos en marzo de 2012, seis de los siete partidos políticos fueron obligados por el IFE a modificarlas para dar cumplimiento a la cuota de género establecida en la ley.

\section{Elecciones subnacionales}

En 2012 también se eligieron seis gobernadores y el jefe de gobierno del Distrito Federal. ${ }^{7}$ En estas contiendas el PRI obtuvo resonantes victorias en Chiapas y en Jalisco, este último gobernado por el PAN desde 1995. El PAN logró retener Guanajuato, un antiguo reducto. Los candidatos del PRD triunfaron en Tabasco, donde el PRI nunca había abandonado el poder, en Morelos, donde desplazó al PAN luego de dos sexenios, y en el Distrito Federal, su tradicional bastión (Ver Tabla 3). A partir de estos resultados, el PRI gobierna veintidós de las treinta y dos entidades; el PAN ocho, el PRD tres y el Distrito Federal, y una alianza conformada por el PAN, PRD y el PT el estado de Oaxaca.

Tabla 3: Resultados de las seis elecciones de gobernador y del jefe de gobierno del Distrito Federal acontecidas en 2012

\begin{tabular}{|c|c|c|c|c|}
\hline Estado & Partido & Candidato & $\begin{array}{c}\% \text { de votación } \\
\text { del ganador }\end{array}$ & Anterior \\
\hline Chiapas & PRI-PVEM & Manuel Velasco Coello & $66 \%$ & PRD \\
\hline Distrito Federal & $\begin{array}{l}\text { PRD-PT-Movimiento } \\
\text { Ciudadano }\end{array}$ & Miguel Ángel Mancera & $64 \%$ & PRD \\
\hline Guanajuato & PAN-Nueva Alianza & Miguel Márquez Márquez & $49 \%$ & PAN \\
\hline Jalisco & PRI-PVEM & Jorge Aristóteles Sandoval Díaz & $40 \%$ & PAN \\
\hline Morelos & $\begin{array}{l}\text { PRD-PT-Movimiento } \\
\text { Ciudadano }\end{array}$ & Graco Ramírez & $43 \%$ & PAN \\
\hline Tabasco & $\begin{array}{l}\text { PRD-PT-Movimiento } \\
\text { Ciudadano }\end{array}$ & Arturo Núñez Jiménez & $50 \%$ & PRI \\
\hline Yucatán & PRI-PVEM-PSD & Rolando Zapata Bello & $33 \%$ & PRI \\
\hline
\end{tabular}

Fuentes: Instituto de Elecciones y Participación Ciudadana de Chiapas, http:/ /www.iepc-chiapas. org.mx/indexnw2.php?modulo=resultados2012

Instituto Electoral del Distrito Federal, http://www.iedf.org.mx/secciones/elecciones/ estadisticas/2012/estadistica.pdf

Instituto Electoral del Estado de Guanajuato, http:/ / www.ieeg.org.mx/

Instituto Electoral y de Participación Ciudadana de Jalisco, http://www.iepcjalisco.org.mx/ resultados-electorales Instituto Estatal Electoral de Morelos, http://www.ieemorelos.org.mx/ Instituto Electoral y de Participación Ciudadana de Tabasco, http:/ / www.iepct.org.mx/ Instituto de Procedimientos Electorales y Participación Ciudadana del Estado de Yucatán, http:/ / www.ipepac.org.mx/resultadosGobernador2012.php

7 En México, las elecciones de gobernadores se dan de manera escalonada en el tiempo a lo largo de cada sexenio presidencial, de modo que solo una porción de las mismas tiene lugar el mismo año que la elección presidencial. 


\section{COYUNTURA SOCIAL}

En el plano social, uno de los temas que marcaron 2012 fue la emergencia de un movimiento estudiantil que de manera vigorosa irrumpió en la escena pública durante la campaña electoral y se convirtió en punto focal hacia el que se canalizó la inquietud y el descontento de quienes veían en una victoria priista la potencial restauración de un pasado autoritario.

Los inicios del movimiento \#Yosoy132 estuvieron ligados a un hecho en principio poco relevante. El 11 de mayo, el candidato del PRI participó en un foro con estudiantes en la Universidad Iberoamericana (una de las universidades privadas más importantes del país) y de modo imprevisto fue recibido con reclamos acerca de sus vínculos con los más oscuros personajes del pasado priista y sus acciones represivas como gobernador del Estado de México. Pasado el incidente, el presidente del PRI acusó a los inconformes de no pertenecer a la universidad y responder a López Obrador. En respuesta, un video en donde 131 estudiantes que habían participado de la protesta mostraban su credencial universitaria fue subido a YouTube y a los pocos días se convirtió en viral (para un análisis detallado ver De Mauleón 2012). Como muestra de apoyo alguien creó el hashtag \#YoSoy132, y Twitter y Facebook hicieron el resto. El grupo inicial (paradójicamente proveniente de universidades privadas de clase media-alta y poco movilizadas políticamente) fue sumando adeptos, y las consignas fueron escalando, denunciando ya no solo el carácter autoritario del PRI, sino la parcialidad y manipulación de la información por parte de los medios de comunicación (en particular, Televisa y TvAzteca, que controlan casi la totalidad de la televisión abierta) en favor de la candidatura de Peña Nieto.

El movimiento adquirió rápidamente un lugar central en la escena pública. En los meses de mayor auge (desde su surgimiento hasta poco después de las elecciones del 1 de julio) sumó adeptos en casi todas las universidades del país y organizó varias movilizaciones (una de las primeras, dirigida a las sedes de Televisa en Ciudad de México). Además, en un hecho inédito, el movimiento promovió con éxito un tercer debate entre los candidatos (adicional a los dos organizados por el IFE) al que asistieron tres de los cuatro postulantes (Peña Nieto fue el ausente) y que se centró en los temas que el movimiento planteaba como sus principales demandas y fue transmitido por internet. A pesar de que algunos de sus voceros adquirieron notoriedad pública, el \#YoSoy132 se caracterizó por la ausencia de líderes, intentando priorizar un carácter horizontal y asambleario. Si en un principio esta cualidad permitió la rápida integración de grupos estudiantiles diversos, a la larga fue también responsable de que una vez pasada la elección el movimiento perdiera fuerza y rumbo e incluso se generaran disputas internas.

También durante 2012 continuó siendo fuerte la presencia del Movimiento por la Paz, con Justicia y Dignidad, surgido en 2011 bajo el liderazgo de Javier Sicilia y comprometido con defensa de los derechos de las víctimas de la "guerra contra el narcotráfico". Por un lado, el movimiento jugó un rol central para la aprobación de la Ley de Víctimas (que se aborda más adelante) y apareció como interlocutor de los candidatos presidenciales, con los que tuvo publicitadas reuniones en las que se les demandó redefinir la estrategia 
de lucha contra el narcotráfico. Por otro lado, se convirtió inclusive en actor durante la campaña presidencial en Estados Unidos, momento durante el cual realizó una caravana por las ciudades más importantes de dicho país demandando leyes de control de armas más estrictas y una redefinición de la política del gobierno norteamericano en relación con la temática de las drogas.

\section{SEGURIDAD PÚBLICA Y VIOLENCIA}

Durante la gestión de Calderón, el combate frontal a las bandas de narcotraficantes fue una de las prioridades de la agenda del presidente, otorgando un rol central a las Fuerzas Armadas en dichas tareas. Tres consecuencias de esta estrategia fueron el incremento de la tasa de homicidios y de la desaparición de personas (en ambos casos principalmente por enfrentamientos entre cárteles) y las violaciones a los derechos humanos por parte de las fuerzas armadas.

De acuerdo con cifras oficiales, el total de muertes por este motivo desde diciembre de 2006 a diciembre de 2011 ha sido de 47.515 (INEGI, 2012). Si los años de 2010 y 2011 fueron los más violentos desde el inicio de esta estrategia, durante 2012 (aun cuando todavía no hay cifras definitivas) la violencia pareció estabilizarse e incluso declinar y su dispersión geográfica se redujo. ${ }^{8}$ Según el Sistema Nacional de Seguridad Pública, el número total de homicidios en 2012 habría sido de entre 20.000 y 25.000 -la cifra en 2011 fue de 27.199- y el número de municipios libres de violencia se habría incrementado en un 16 por ciento (Molzahn, Rodríguez y Shirk, 2013). De acuerdo con Guerrero Gutiérrez (2012), la estabilización o posible declinación de la violencia se explicaría por cuatro factores: las dificultades de las organizaciones criminales para reclutar sicarios debido a los riesgos de la actividad, el surgimiento de movimientos sociales que comenzaron a denunciar las violaciones a los derechos humanos por parte de los cuerpos de seguridad pública, respuestas más oportunas a los brotes de violencia, y la decisión del gobierno federal de focalizar los esfuerzos en las organizaciones criminales más violentas.

En el marco de su intervención en actividades de seguridad pública, las Fuerzas Armadas han incurrido en graves violaciones a los derechos humanos. De acuerdo con Human Rights Watch, la Procuraduría General de Justicia Militar inició cerca de cinco mil investigaciones sobre violaciones de garantías a civiles cometidas por miembros del ejército entre 2007 y principios de 2012, pero en ese período solamente fueron condenados por tales hechos treinta y ocho soldados.

Estas violaciones a los derechos humanos han sido investigadas y juzgadas en la jurisdicción militar (Human Rights Watch, 2011), pero a partir de decisiones de la Corte Interamericana de Derechos Humanos y de la Suprema Corte Justicia mexicana esto ha comenzado a cambiar. En septiembre de 2012 se alcanzó un hito cuando la Suprema

8 En noviembre de 2012, el gobierno anunció que dejaría de reportar los homicidios relacionados con la delincuencia organizada -argumentando que este tipo de violencia no está codificado por la ley y por lo tanto recopilar la información relevante es difícil. La base de datos sobre "fallecimientos por presunta rivalidad delincuencial" se discontinuó y ello impide usarla para analizar tendencias (Guerrero Gutiérrez, 2012). 
Corte acotó de manera crucial y definitiva el fuero militar declarando inconstitucional el Artículo 57, Fracción II, Inciso "A" del Código de Justicia Militar y determinando que un militar que cometa cualquier delito contra un civil deberá ser juzgado por tribunales del fuero común. Además, la Suprema Corte estableció que en los casos de homicidio o desapariciones forzadas cometidos por militares, los familiares también son víctimas y tienen el derecho de exigir reparación y de impugnar sentencias. Como consecuencia de este fallo, al concluir 2012 los cuatro casos de abusos cometidos por militares sobre los que la Corte Interamericana había dictado sentencia fueron transferidos a la jurisdicción civil. Sin embargo, esos casos fueron los únicos; el resto de las violaciones de derechos humanos continuó bajo la jurisdicción militar (Amnesty International, 2012).

En relación con las desapariciones forzadas o involuntarias, en marzo de 2012 se presentó en Ginebra el informe del Grupo de Trabajo de Naciones Unidas sobre las Desapariciones Forzadas o Involuntarias (GTDFI), que denunció la ausencia de esfuerzos para determinar el número y el destino de las personas desaparecidas y para sancionar a los culpables (GTDFI, 2012). ${ }^{9}$ La aprobación, en marzo de 2012, de la Ley del Registro Nacional de Datos de Personas Extraviadas o Desaparecidas constituyó un avance en la sistematización de la información sobre desapariciones y el seguimiento de las denuncias, creando el registro de personas desaparecidas. Hasta entonces, la cifra respecto a desaparecidos dependía de la fuente. La Secretaría de Seguridad Pública federal (SSP) tenía registradas alrededor de dos mil personas desaparecidas, la Procuraduría General de la República (PGR) 4.800 casos y la Comisión Nacional de los Derechos Humanos más de cinco mil entre 2006 y $2011 .{ }^{10} \mathrm{~A}$ fines de 2012 ganó estado público la denominada "Base integrada de personas no localizadas" presuntamente como resultado de una filtración desde la PGR registrando 20.851 personas desaparecidas entre 2006 y 2012, siendo 2010 y 2011 los años con más desaparecidos.

\section{COYUNTURA INTERNACIONAL}

En el plano internacional, uno de los acontecimientos que mayor expectativa género en México durante 2012 fue la elección presidencial en los Estados Unidos, que además coincidió con la mexicana luego de 12 años.

Paradójicamente, la relación bilateral con México ocupó un lugar marginal durante la campaña estadounidense y casi no fue mencionada en los debates entre los candidatos. Sin embargo, dos temas adquirieron particular relevancia para México: la cuestión migratoria y la temática de las drogas. Con respecto al primero, y aunque no fue

$9 \quad$ El informe emitió treinta y cuatro recomendaciones, entre las que se pueden mencionar la creación de un Programa de Búsqueda de Desaparecidos, la aplicación de protocolos de uso de la fuerza, reforma al fuero militar y regreso de los militares a los cuarteles, revelación de los nombres de los responsables de las desapariciones forzadas durante la "Guerra Sucia" -recabados por la extinta Fiscalía Especializada para Movimientos Sociales y Políticos del Pasado- y reparación del daño a los familiares de víctimas.

10 En mayo de 2012 se realizó la "Marcha de la dignidad nacional: madres buscando a sus hijos e hijas" para demandar del gobierno mexicano que acate las recomendaciones del GTDFI de la ONU. 
un eje central de su agenda, el candidato republicano Romney mostró en diversas oportunidades signos de que en caso de ser elegido endurecería las regulaciones y sanciones a los inmigrantes indocumentados. En contraste, y a contramano de lo que fue norma durante todo su primer mandato, Obama lanzó una serie de iniciativas para suspender las deportaciones de cierto tipo de inmigrantes y garantizar su estadía en suelo estadounidense, mientras dejaba entrever que impulsaría una reforma migratoria más integral en caso de ser reelegido.

En relación con el segundo punto, la aprobación de la legalización de la marihuana para uso recreativo, en referéndums llevados a cabo en los estados de Washington y Colorado, podría tener un profundo impacto en las actividades de los carteles mexicanos al tiempo que quitaría legitimidad a las presiones del gobierno estadounidense para sostener las acciones contra el crimen organizado (Hope y Clark, 2012).

La victoria de Obama parece haber abierto la puerta para avanzar en el tema migratorio, y esto podría generar cambios en la relación bilateral. Asimismo, el entrante gobierno de Peña Nieto parece haber decidido darle más peso a lo económico en el vínculo entre ambos países, quitando centralidad a la lucha contra el crimen organizado. ${ }^{11}$

También, durante 2012 un confuso hecho volvió a confirmar la presencia activa en México de agentes estadounidenses involucrados en el adiestramiento y la capacitación de las fuerzas de seguridad mexicanas, y la penetración que el crimen organizado tiene en las mismas. En agosto, un automóvil diplomático que transportaba a dos agentes estadounidenses fue atacado por agentes de la Policía Federal. Si bien los agresores fueron detenidos, las investigaciones acerca de los motivos del ataque, así como las tareas en las que estaban involucrados los norteamericanos nunca se hicieron públicas, al tiempo que la interacción entre ambos gobiernos en torno al hecho se llevó adelante por canales reservados. ${ }^{12}$

\section{LA ECONOMÍA MEXICANA}

Durante 2012 la economía mexicana continuó creciendo moderadamente tal como ya se había observado en años anteriores y en línea con la recuperación posterior a la crisis de 2009. El incremento interanual del PIB fue de 3,9\%, similar al valor registrado en 2011. ${ }^{13}$ El Indicador Global de la Actividad Económica (IGAE) del Instituto Nacional de Estadística y Geografía (INEGI) revela que el mayor dinamismo de la economía tuvo lugar durante la primera mitad del año.

En relación con el empleo, al tercer trimestre de 2012 la desocupación se encontraba en un 5,15\%, cifra algo mayor que la registrada en los primeros dos trimestres del año, pero dentro de los parámetros recientes. Asimismo, la balanza comercial volvió a mostrar

11 Revista Proceso, "Pretenden Peña Nieto y Obama desnarcotizar la relación bilateral" 27 de noviembre de 2012. http:/ / www.proceso.com.mx/?p=326350

12 Milenio, “Dos empleados de EU heridos tras balacera en Tres Marías" 24 de agosto de 2012. http:/ /www. milenio.com/cdb/doc/noticias2011/2d68a40be602f9524eeed782ad87806b

13 INEGI (2013). 
un saldo negativo, ubicándose el déficit en alrededor de USD 800 millones. Durante el período enero-noviembre 2012 las exportaciones crecieron un 6,4\% respecto del mismo período durante el año anterior, mientras que las importaciones se incrementaron en un $6,2 \%$. En relación con las primeras, el punto más destacado fue el aumento de las exportaciones no petroleras (que tuvieron un aumento interanual de 8,5\%), al tiempo que las exportaciones petroleras mostraron una variación interanual negativa, ya que cayeron un $4,6 \% .^{14}$

La estabilidad macroeconómica que desde hace tiempo caracteriza a México, no se vio amenazada durante 2012 por los vaivenes políticos del proceso electoral ni por la perspectiva de alternancia en el partido en la presidencia. No obstante ello, algunas variables registraron altibajos a lo largo del año.

Aunque la tasa anual de la inflación se ubicó al finalizar 2012 en un 3,57\%, valor dentro del rango de las metas fijadas por el Banco de México, durante buena parte del año se situó ligeramente por sobre el tope del $4 \%$ establecido como meta por dicha entidad. Esta situación llevó a su presidente, Agustín Carstens, a declarar públicamente que el Banco de México intervendría activamente utilizando herramientas de política monetaria para corregir dicha situación. ${ }^{15}$

Por último, el tipo de cambio cerró el año con un valor apenas inferior al registrado a fines de 2011. Un ligero incremento fue observado particularmente durante el período abril-junio, explicado en gran medida por las turbulencias derivadas de la crisis europea, pero que algunos analistas (quizás intencionadamente) también atribuyeron al crecimiento en la intención de voto por López Obrador en las vísperas de las elecciones presidenciales de julio. ${ }^{16}$

\section{CAMBIOS INSTITUCIONALES Y REFORMAS LEGISLATIVAS}

El 2012 fue un año particularmente significativo en lo que respecta a reformas legislativas con impacto en el entramado constitucional e institucional.

En agosto de 2012, después de dos años de discusión, se publicó la reforma política. Entre sus puntos destacados aparecen la incorporación de las candidaturas independientes, la creación de dos mecanismos de democracia directa -iniciativa popular y consulta popular-, la erradicación de la sobrerrepresentación partidaria en la Asamblea Legislativa del Distrito Federal y la iniciativa presidencial preferente -o de trámite urgente. Esta última faculta al Presidente a presentar hasta dos iniciativas el día de la apertura de cada periodo ordinario de sesiones del Congreso (o en su defecto a señalar con tal carácter hasta dos iniciativas presentadas en periodos anteriores) que deben ser tratadas por el Legislativo en un plazo de sesenta días.

15 El Economista, "Banxico intervendría para bajar inflación: Carstens". 30 de Octubre de 2012. http:/ / eleconomista. com.mx/finanzas-publicas/2012/10/30/inflacion-ya-estaria-afectando-salarios-carstens

16 Animal Político, “Caída de Peña presiona al peso: analistas de Reuters". 31 de Mayo de 2012. http://www. animalpolitico.com/2012/05/caida-de-pena-presiona-al-peso/ 
Haciendo uso de esta nueva facultad, Calderón envió en septiembre dos iniciativas preferentes al Congreso: las propuestas de reforma a la Ley General del Trabajo y a la Ley General de Contabilidad Gubernamental. ${ }^{17}$ La reforma laboral se centró en la flexibilización de los esquemas de contratación y en mecanismos para promover la democracia y la transparencia sindical (por ejemplo, propiciando la elección de los dirigentes sindicales mediante voto secreto y haciendo voluntarios los aportes de los afiliados). Estos últimos puntos generaron conflictos con los sectores del PRI ligados a los sindicatos y, si bien el PRD y el PAN en el Senado insistieron en ellos, en la Cámara de Diputados la fracción del PAN apoyó al PRI en una versión diluida que evitara el rechazo a la reforma. ${ }^{18}$ Finalmente, el presidente Calderón logró promulgar la nueva legislación laboral en el último día de su mandato. Por su parte y la reforma a la Ley General de Contabilidad Gubernamental -apoyada por todo el espectro político excepto los senadores del Partido del Trabajo- incorporó mecanismos para transparentar la información financiera sobre el ejercicio de los recursos públicos en los tres órdenes de gobierno: municipal, estatal y federal.

En otro orden, el 30 de abril de 2012 el Congreso aprobó la Ley de Víctimas y determinó su publicación en el Diario Oficial de la Federación para el 30 de junio. El proyecto original fue propuesto por expertos del Instituto Nacional de Ciencias Penales y de la UNAM, impulsado por las víctimas movilizadas en torno al Movimiento por la Paz con Justicia y Dignidad, y aprobado por unanimidad en ambas cámaras. La norma sancionada reconoce y garantiza los derechos de las víctimas del delito y de violaciones a derechos humanos, estableciendo entre otros el derecho a la asistencia, protección, atención, verdad, justicia, reparación integral, restitución de sus derechos violados, debida diligencia y no repetición. Sin embargo, desde el gobierno de Calderón fue objeto de críticas por su inviabilidad y por deficiencias de técnica legislativa y el presidente promovió una controversia constitucional y no la promulgó. ${ }^{19}$ A su llegada a la presidencia, Peña Nieto se comprometió a retirar la controversia constitucional y a promulgarla.

Finalmente, al llegar al gobierno Peña Nieto promovió una reforma educativa que recibió el apoyo de las principales fuerzas políticas y de amplios sectores de la sociedad orientada a limitar la influencia del Sindicato Nacional de Trabajadores de la Educación, limitar prácticas como la venta y/o transmisión hereditaria de plazas de maestro y promover la transparencia. La misma fue aprobada por el Congreso de la Unión en los últimos días de diciembre. A partir de esta reforma se crea el servicio profesional docente estableciendo "la evaluación obligatoria para el ingreso, la promoción, el reconocimiento

17 En México los nuevos miembros del Congreso asumen sus cargos en septiembre, pero los presidentes toman protesta en diciembre. Por consiguiente, entre septiembre y noviembre de 2012, el presidente Calderón convivió con un Congreso renovado de acuerdo con los resultados del 1 de julio y con un nuevo presidente elegido. Dadas estas circunstancias, las iniciativas preferentes habrían sido acordadas entre el entonces presidente en ejercicio y el presidente entrante.

18 Animal Político, "El PRI impone su Reforma Laboral". 9 de noviembre de 2012. http:/ / www.animalpolitico. com/2012/11/el-pri-impone-su-reforma-laboral/\#axzz2OKetDi8S

19 La Jornada. “La ley de víctimas, bastante malita; hay que hacer otra, dice Soberanes” 15 de enero de 2013. http://www.jornada.unam.mx/2013/01/15/politica/005n2pol\#sthash.cHfm6NQx.dpuf 
y la permanencia en el servicio profesional", un Sistema Nacional de Evaluación bajo la responsabilidad del Instituto Nacional de Evaluación de la Educación, y un Sistema Nacional Único de Información Educativa.

\section{PODER EJECUTIVO}

El 2012 fue un año trascendente en lo que respecta al poder ejecutivo dada la finalización del mandato de Calderón y la llegada a la presidencia de Peña Nieto. Si bien Calderón ostentó el cargo hasta el 1 de diciembre de 2012, durante el último año de su gobierno su capacidad para impulsar nuevas políticas y/o instalar temas en la agenda se vio limitada por una serie de factores. En primer lugar, por las restricciones legales a las acciones del Ejecutivo durante la campaña electoral para evitar que el presidente use los actos de gobierno para favorecer a alguno de los candidatos. En segundo lugar, porque luego de la victoria de Peña Nieto la atención se centró en la agenda del presidente entrante. En tercer lugar, porque como ya se ha explicado (ver nota 19) Calderón debió convivir entre septiembre y diciembre con el Congreso elegido en julio, en el que el PAN tiene menor representación. Así, si bien Calderón impulsó durante la segunda parte del año una serie de reformas por las que había abogado en el pasado, el contenido de las mismas pareció resultar de negociaciones con el presidente elegido y su equipo.

Sin duda, la "inacción" de Calderón durante su último año contrastó significativamente con la hiperactividad de las semanas que siguieron a la asunción de Peña Nieto el 1 de diciembre de 2012, y las acciones desarrolladas por el presidente entrante en pos de comenzar a materializar su agenda reformista.

En su primer discurso como presidente, Peña Nieto enumeró trece líneas concretas de acción, algunas de aplicación inmediata. En materia de seguridad pública el nuevo mandatario propuso el diseño de un programa nacional de prevención del delito en manos de las secretarías de Gobernación, Hacienda, Salud, Desarrollo Social y de Educación; la propuesta al Congreso para la simplificación de los códigos penales y del ejército, y la publicación de la ley de víctimas. ${ }^{20}$ En políticas de combate a la pobreza, las decisiones fueron impulsar una cruzada nacional contra el hambre, la creación de un seguro de vida para jefas de familia y la ampliación del programa "Setenta y más" (que otorgaba una pensión mensual universal a los mayores de setenta años) para incluir a los mayores de sesenta y cinco años. En el mediano plazo, todas estas iniciativas deberían concatenarse para avanzar hacia la consolidación de un sistema universal de seguridad social. En materia de educación propuso la reforma para crear el servicio profesional de carrera magisterial. En el rubro infraestructura y telecomunicaciones la recuperación del servicio de trenes, programas de infraestructura para conectar el sur de México e iniciativas para incrementar la competencia en telefonía, televisión y radio. Por 
último, en materia económica y financiera propuso la ley nacional de responsabilidad hacendaria para regular el endeudamiento de los gobiernos subnacionales y medidas de austeridad y disciplina fiscal.

Asimismo, y en línea con los diagnósticos que durante los últimos años se propusieron desde ciertos organismos internacionales (muy claramente desde la OCDE, de la que México es miembro) y desde sectores influyentes dentro de México, Peña Nieto propuso una agenda de reformas estructurales orientadas a potenciar la competitividad y promover un mayor crecimiento. El inicio del nuevo gobierno estuvo definido no solo por la centralidad de esta agenda, sino por la decisión de consensuarla con los otros partidos con el fin de materializarla. Este acuerdo se plasmó en lo que se dio en llamar el "Pacto por México", un documento negociado durante los meses de la transición y finalmente suscrito por Peña Nieto y las dirigencias del PAN y el PRD en un publicitado evento, del que participaron importantes actores políticos, sociales y empresariales. ${ }^{21}$ En el "Pacto" se acordaron tanto un listado de reformas consideradas necesarias para el país como un calendario para su tratamiento, así como el compromiso de los partidos a brindar apoyo legislativo. ${ }^{22}$ La sanción de una reforma educativa (mencionada en la sección anterior) fue el primer fruto concreto de este acuerdo.

\section{Conformación y funcionamiento del gabinete}

Dado que durante 2012 no se registraron variaciones significativas en el gabinete de Calderón, ${ }^{23}$ en lo que sigue nos concentramos en el análisis del gabinete de Peña Nieto.

En la elección de los miembros de su gabinete el presidente entrante pareció privilegiar tanto la confianza personal como las capacidades políticas de los funcionarios en puestos clave para negociar con los actores relevantes en cada sector. Las Secretarías más importantes fueron ocupadas por personalidades con amplia trayectoria en el PRI y en muchos casos con larga experiencia en funciones ejecutivas en el gobierno federal o en gobiernos estatales y locales (por ejemplo, en el gabinete hay cinco exgobernadores), aliados políticos de Peña Nieto y miembros de su equipo más cercano durante su mandato como gobernador del Estado de México (ver lista de miembros en Tabla 4). Puede especularse que la priorización de este tipo de perfiles obedece al objetivo de la nueva administración de impulsar una serie de reformas que en el

Los motivos de las autoridades del PAN y del PRD para adherir al Pacto parecen haber sido diversos. En el caso del PAN, parece existir coincidencia programática con el contenido de las reformas propuestas, algunas de las cuales fueron promovidas sin éxito por Fox y Calderón. El apoyo del PRD probablemente se explique por la intención de los sectores moderados que controlan al partido de consolidar una imagen dialoguista y "racional" que consideran necesaria para recuperar el apoyo electoral de las clases medias. En cualquier caso, la adhesión al Pacto ha sido cuestionada y criticada por diversos sectores al interior de ambos partidos.

22 El documento puede consultarse en http://pactopormexico.org/.

23 Durante 2012 el único cambio en el gabinete de Calderón se dio en la Secretaría de Educación Pública (SEP) que quedó vacante en abril a partir de la renuncia de Alonso Lujambio por motivos de salud y hasta el final de la administración fue ocupada por José Ángel Córdova Villalobos (quien había sido Secretario de Salud). 
Tabla 4: $\quad$ Gabinete nombrado por Enrique Peña Nieto (1 de diciembre de 2012)

\begin{tabular}{|c|c|c|c|}
\hline Dependencia & Titular & $\begin{array}{c}\text { Formación } \\
\text { (último grado de estudios) }\end{array}$ & $\begin{array}{l}\text { Partido } \\
\text { político }\end{array}$ \\
\hline Secretaría de Gobernación & Miguel Ángel Osorio Chong & Licenciado en Derecho & PRI \\
\hline $\begin{array}{l}\text { Secretaría de Hacienda y } \\
\text { Crédito Público }\end{array}$ & Luis Videgaray & Doctor en Economía & PRI \\
\hline Secretaría de Energía & Pedro Joaquín Coldwell & Licenciado en Derecho & PRI \\
\hline Secretaría de Desarrollo Social & Rosario Robles & Maestra en Desarrollo Rural & $\begin{array}{l}\text { Ex PRD, ahora } \\
\text { independiente }\end{array}$ \\
\hline Secretaría de Educación Pública & Emilio Chuayfett & Licenciado en Derecho & PRI \\
\hline $\begin{array}{l}\text { Secretaría de Comunicaciones } \\
\text { y Transporte }\end{array}$ & Gerardo Ruiz Esparza & Maestro en Derecho & PRI \\
\hline Secretaría de Economía & Idelfonso Guajardo & $\begin{array}{l}\text { Doctor en Finanzas Públicas y } \\
\text { Economía }\end{array}$ & PRI \\
\hline $\begin{array}{l}\text { Secretaría de Relaciones } \\
\text { Exteriores }\end{array}$ & José Antonio Meade & Doctor en Economía & Independiente \\
\hline $\begin{array}{l}\text { Secretaría de la Defensa } \\
\text { Nacional }\end{array}$ & Salvador Cienfuegos Zepeda & $\begin{array}{l}\text { Maestro en Administración } \\
\text { Militar para la Seguridad y } \\
\text { Defensa Nacionales }\end{array}$ & Independiente \\
\hline Secretaría de la Marina & Vidal Soberón & $\begin{array}{l}\text { Licenciado en Ingeniería } \\
\text { Mecánica Naval }\end{array}$ & Independiente \\
\hline $\begin{array}{l}\text { Secretaría de Medio Ambiente } \\
\text { y Recursos Naturales }\end{array}$ & Juan José Guerra & Maestro en Economía & PRI \\
\hline $\begin{array}{l}\text { Secretaría de Agricultura, } \\
\text { Ganadería, Desarrollo Rural, } \\
\text { Pesca y Alimentación }\end{array}$ & Enrique Martínez y Martínez & Licenciado en Economía & PRI \\
\hline Secretaría de Salud & Mercedes Juan López & Medica Cirujana & PRI \\
\hline $\begin{array}{l}\text { Secretaría de Trabajo y } \\
\text { Previsión Social }\end{array}$ & Alfonso Navarrete Prida & Maestro en Derecho Penal & PRI \\
\hline Secretaría de la Reforma Agraria & Jorge Carlos Ramírez Marín & Licenciado en Derecho & PRI \\
\hline Secretaría de Turismo & Claudia Ruiz Massieu & $\begin{array}{l}\text { Candidata a Doctor en } \\
\text { Derecho Público y Filosofía } \\
\text { Jurídica }\end{array}$ & PRI \\
\hline $\begin{array}{l}\text { Consejero Jurídico de la } \\
\text { Presidencia }\end{array}$ & Humberto Castillejos & Licenciado en Derecho & PRI \\
\hline $\begin{array}{l}\text { Procuraduría General } \\
\text { de la República }\end{array}$ & Jesús Murillo Karam & Licenciado en Derecho & PRI \\
\hline
\end{tabular}

Fuente: Presidencia de la República http:/ / www.presidencia.gob.mx/gabinete/ 
pasado generaron resistencias sustantivas (como por ejemplo en las áreas de energía y educación).

Las únicas secretarías no ocupadas por priistas (además de las de Defensa y Marina, históricamente encomendadas a militares) fueron la de Desarrollo Social y la de Relaciones Exteriores. La primera se encomendó a Rosario Robles, en su momento presidenta del PRD y jefa de gobierno interina del Distrito Federal, quien ya desde la campaña se sumó a Peña Nieto para articular la relación con ciertos sectores sociales. Para la segunda se designó a quien hasta el final del gobierno de Calderón se desempeñara como Secretario de Hacienda, José Antonio Meade, confirmando que el nuevo gobierno piensa encarar las relaciones internacionales (con Estados Unidos y también con el resto del mundo) enfatizando el eje económico/comercial y no tanto la agenda de la lucha contra el narcotráfico. Por último, resultó también inesperada la nominación de Manuel Mondragón y Kalb a un puesto estratégico en el área de seguridad (poniendo bajo su responsabilidad la Policía Federal) dado que durante el período 2006-2012 había sido un funcionario central del gobierno perredista de la Ciudad de México. Es importante destacar que dichas designaciones no se inscribieron en un acuerdo entre Peña Nieto y las otras fuerzas políticas con el objetivo de darle pluralidad al nuevo gabinete y/o garantizar el apoyo de las mismas a las acciones de gobierno, sino más bien al reconocimiento por parte del presidente de las capacidades técnicas de los nombrados y/o a los vínculos personales con el nuevo mandatario y miembros de su equipo.

En su funcionamiento y organización, el nuevo gabinete se caracteriza por la existencia de dos "hombres fuertes": el Secretario de Hacienda y Crédito Público, Luis Videgaray, y el Secretario de Gobernación, Miguel Ángel Osorio Chong, ambos con relación de larga data con Peña Nieto y operadores centrales durante su campaña. El poder de Osorio Chong resulta en gran medida de la decisión de concentrar bajo su mando la responsabilidad de la seguridad pública (y el control de las fuerzas de seguridad como la Policía Federal), volviendo a la tradición de los gobiernos priistas del pasado. Dicha redefinición de funciones supuso para el nuevo presidente la necesidad de promover una reforma a la Ley Orgánica de la Administración Pública Federal orientada a disolver la Secretaría de Seguridad Pública (creada durante el gobierno de Fox justamente para separar las tareas de seguridad de las desempeñadas por la Secretaría de Gobernación -SEGOB-) y promover la incorporación de sus responsabilidades nuevamente a la SEGOB. ${ }^{24}$

Si bien se esperaba una rápida ratificación de la reforma, su tratamiento y aprobación generaron mayores resistencias de las previstas. Una alianza entre el PAN y el PRD en el Senado logró introducir modificaciones importantes, instituyendo la obligación para que el comisionado nacional de Seguridad y el secretario Ejecutivo del Sistema de

24 El proyecto de redefinición de la estructura del gobierno federal suponía además la disolución de la Secretaría de la Función Pública (otra creación de Fox) y su reemplazo por un órgano independiente responsable del combate a la corrupción. Esta reforma todavía no se ha materializado. 
Seguridad Pública (que coordina la acción del gobierno federal y los gobiernos estatales) deban contar con ratificación del Congreso para asumir sus funciones. ${ }^{25}$

\section{PODER LEGISLATIVO}

En la legislatura que se instaló el 1 de septiembre de 2012, el PRI logró conformar los bloques más numerosos en ambas cámaras. En Diputados alcanzó un 42 por ciento de los escaños, pero redujo su participación con respecto a la legislatura anterior en casi seis puntos porcentuales. Esta distribución obliga al nuevo presidente a buscar apoyo en otras fuerzas políticas, tales como el Partido Verde -parte de la coalición electoral que apoyó a Peña Nieto y por lo tanto aliado seguro- y Nueva Alianza. En el Senado, en cambio, el PRI pasó de tener poco más de un cuarto de la Cámara a contar con el 40 por ciento de los Senadores.

Un hecho importante es que a partir de esta nueva composición por primera vez el PAN quedó relegado a ser la tercera fuerza y la alianza de partidos de izquierda -PRD, PT y Movimiento Ciudadano- alcanzó la mayor representación en la historia (Ver Tabla 5).

Tabla 5: Cámaras de Diputados y de Senadores: Composición por grupo parlamentario

\begin{tabular}{|c|c|c|c|c|c|c|c|c|}
\hline & \multicolumn{4}{|c|}{ Cámara de Diputados } & \multicolumn{4}{|c|}{ Senado } \\
\hline & \multicolumn{2}{|c|}{$2009-2012$} & \multicolumn{2}{|c|}{ 2012-2015 } & \multicolumn{2}{|c|}{ 2006-2012 } & \multicolumn{2}{|c|}{$2012-2018$} \\
\hline & & $\%$ & & $\%$ & & $\%$ & & $\%$ \\
\hline PAN & 142 & 28,40 & 114 & 22,8 & 52 & 40,62 & 38 & 29,68 \\
\hline PRI & 242 & 48,40 & 213 & 42,6 & 33 & 25,78 & 52 & 40,62 \\
\hline PRD & 63 & 12,60 & 104 & 20,8 & 26 & 20,31 & 22 & 17,18 \\
\hline PVEM & 22 & 4,40 & 28 & 5,6 & 6 & 4,68 & 9 & 7,03 \\
\hline $\mathrm{MC}$ & 60 & 1,20 & 16 & 3,2 & & & 2 & 1,56 \\
\hline PT & 14 & 2,80 & 15 & 3 & 4 & 3,12 & 4 & 3,12 \\
\hline Nueva Alianza & 8 & 1,60 & 10 & 2 & 1 & 0,78 & 1 & 0,78 \\
\hline Independiente & 3 & 0,60 & 0 & 0 & 0 & 0 & 0 & 0 \\
\hline Convergencia & 0 & 0 & 0 & 0 & 6 & 4,68 & 0 & 0 \\
\hline Total & 500 & 100 & 500 & 100 & 128 & 100 & 128 & 100 \\
\hline
\end{tabular}

Fuentes: Cámara de Diputados http://sitl.diputados.gob.mx/LXII_leg/info_diputados.php Senado http://www.senado.gob.mx/

25 Este punto fue resistido por los senadores priistas, quienes finalmente retiraron sus reservas con la finalidad de lograr la aprobación de la reforma en lo general. 


\section{PODER JUDICIAL}

Durante los últimos meses del mandato de Calderón se completaron dos vacantes en la Suprema Corte de Justicia de la Nación (SCJN) generadas a raíz de la salida de los magistrados Salvador Aguirre Anguiano y Guillermo Ortiz Mayagoitia. El proceso se llevó a cabo luego de que asumieran los nuevos senadores el 1 de septiembre, lo que explica que en una primera ronda las ternas enviadas por el presidente fuesen rechazadas (generando cierta rispidez entre Calderón y el Legislativo) y que las siguientes debieran ser consensuadas con Peña Nieto y su equipo. En la segunda vuelta el Senado finalmente eligió de los postulantes propuestos a Alfredo Gutiérrez Ortiz Mena (hasta entonces jefe del Sistema de Administración Tributaria-SAT) y a Alberto Gelacio Pérez Dayán, hasta entonces magistrado de un tribunal colegiado en materia administrativa.

\section{CONCLUSIONES Y PERSPECTIVA}

Como hemos mostrado a lo largo de este artículo, 2012 fue un año de eventos importantes en la vida de México. Los resultados en las elecciones de julio y el regreso del PRI a la presidencia luego de 12 años de gobiernos panistas fueron los hechos más trascendentes, dados los efectos sustantivos en la realidad nacional derivados de este cambio.

En 2013 podrán comenzar a vislumbrarse más claramente los primeros resultados de la agenda del nuevo presidente. También será cuando comience a quedar en claro el posible sello distintivo del mandato de Peña Nieto, que seguramente marcará el resto de su sexenio. Si durante el primer año de Fox comenzó a comprenderse que las promesas y expectativas asociadas con la alternancia no se traducirían fácilmente en cambios sustantivos, y en el inicio del gobierno de Calderón se reveló la centralidad que el entonces presidente otorgaba en su agenda de gobierno a la "guerra contra el narcotráfico", los primeros meses del nuevo gobierno priista parecen haber puesto el eje en la concretización de una serie de reformas que se proponen como estructurales.

La forma en que Peña Nieto atienda dos desafíos marcará las perspectivas para el resto de su mandato. En primer lugar, el control y la reducción de la violencia. A pesar de que el crecimiento sostenido de homicidios y ejecuciones pareció detenerse en 2012, las cifras continúan siendo preocupantes. Si bien la administración entrante ha dejado en claro que propone un cambio de estrategia de seguridad, centrándose en los delitos de alto impacto antes que en el combate frontal a los cárteles, reduciendo la centralidad que se le otorgó al tema durante la anterior administración y retirando paulatinamente al Ejército de tareas relacionadas con la seguridad interna, los resultados de este cambio están aún por verse. Por ejemplo, las cifras de ejecutados por el crimen organizado no han experimentado una reducción significativa durante los primeros meses de la nueva administración. En segundo lugar, el gobierno de Peña Nieto enfrenta otro desafío sustantivo en el plano económico. Si durante los últimos años México logró recuperarse de la crisis financiera internacional de 2009 y retomó una senda de crecimiento moderado, el nuevo presidente se ha propuesto generar las condiciones para que México pueda crecer a ritmos más elevados. Desde diversos medios se ha señalado que la evolución 
de ciertos factores del contexto internacional (como por ejemplo el aumento de los salarios en China y la desaceleración de Brasil, junto con el incremento en los costos de logística y transporte) pone a México en una situación ventajosa para potenciar su rol como exportador de productos manufacturados con valor agregado, principalmente hacia el mercado norteamericano. ${ }^{26}$ Las políticas del nuevo gobierno deberían lograr que la economía mexicana pueda sacar provecho de esta situación.

El interrogante estará dado por ver hasta qué punto el nuevo presidente y su partido puedan lograr resultados sin caer en las prácticas autoritarias del pasado y asegurando que las reformas tengan un impacto directo en los amplios sectores de la población que continúan viviendo en condiciones de rezago social. De cómo se resuelvan estos desafíos depende en gran medida el futuro de México.

\section{REFERENCIAS}

CIC. 2012. Informe CIC personas desparecidas. Centro de Investigación y Capacitación Propuesta Cívica. Disponible en http:/ / desaparecidosenmexico.wordpress.com.

Consulta Mitofsky. 2006. México: Índice de confianza en instituciones. Agosto 2006. México DF.

Consulta Mitofsky. 2012. México: confianza en instituciones. Agosto 2012. México DF.

De Mauleón, Héctor. 2012. "De la Red a las calles". Revista Nexos, 1 de septiembre de 2012.

Flores-Macías, Gustavo. 2013. "Mexicos's 2012 Elections: The Reurn of the PRI". Journal of Democracy 24 (1): 128-141.

Fuentes Berain Villenave, Rossana María. 2012. “3 meses de campaña presidencial narrados por 10 encuestas" ADN Político, http://www.adnpolitico.com/encuestas/2012/06/27/3 -meses-de-campana-presidencial-contados-por-10-encuestas

Guerrero Gutiérrez, Eduardo. 2012. La estrategia fallida, Nexos 1 de diciembre de 2012.

GTDFI. 2012. Informe de Misión a México, Grupo de Trabajo de la ONU sobre las Desapariciones Forzadas o Involuntarias.

Hope, Alejandro y Eduardo Clark. 2012. Si los vecinos legalizan. Reporte Técnico. Ciudad de México, IMCO.

INEGI. 2012. "Información oportuna sobre la balanza comercial de mercancías de México durante noviembre de 2012". Boletín de prensa, núm. 458/12.

INEGI. 2013. "Producto Interno Bruto en México durante el cuarto trimestre de 2012". Boletín de prensa, núm. 077/13.

Magaloni, Beatriz, Alberto Díaz-Cayeros, Jorge Olarte y Edgar Franco Vivanco. 2012. La geografía electoral de 2012. México: México Evalúa.

Molzahn, Cory, Octavio Rodríguez Ferreira y David A. Shirk. 2013. Drug Violence in Mexico: Data and Analysis Through 2012. San Diego, Trans-Border Institute, University of San Diego.

O’Neil, Shannon K. y Alejandro Moreno. 2012. "México's Democratic Malaise" Blog Latin America's Moment, Council of Foreign Relations. http:/ /blogs.cfr.org/oneil/2013/02/01/mexicos-democraticmalaise/?utm_source=feedburner\&utm_medium=feed\&utm_campaign=Feed $\% 3 \mathrm{~A}+$ soneil+\%28Sh annon+K.+O\%27Neil\%3A+Latin+America $\% 27$ s+Moment $\% 29$

Suprema Corte de Justicia de la Nación. 2011. Semanario Judicial de la Federación y su Gaceta, Libro III, Diciembre de 2011, Tomo 1. Tesis P. LXXI/2011 (9a.).

26 En noviembre de 2012, pocos días antes del inicio de la presidencia de Peña Nieto, la revista "The Economist" dedicó su portada a México bajo el título "The rise of Mexico" ("El ascenso de México") e incluyó un reporte especial centrado en las potencialidades de la economía mexicana. http://www.economist.com/news/ leaders / 21567081-america-needs-look-again-its-increasingly-important-neighbour-rise-mexico 
Juan C. Olmeda. PhD en Ciencia Política, Northwestern University. Coordinador de Consultorías en la Escuela de Administración Pública del Distrito Federal. (Las opiniones vertidas por el autor son de carácter personal y no reflejan las posturas de esta institución).

E-mail: jcolmeda@gmail.com

María Alejandra Almesto. PhD en Ciencia Política, University of Notre Dame. Departamento de Ciencias Sociales, Universidad Autónoma Metropolitana, Unidad Cuajimalpa.

E-mail: alejandra.armesto@gmail.com 
